\title{
Modulation of Heparin Cofactor II Activity by Histidine-rich Glycoprotein and Platelet Factor 4
}

\author{
Douglas M. Tollefsen and Cynthia A. Pestka \\ Division of Hematology-Oncology, Departments of Internal Medicine and Biological Chemistry, \\ Washington University School of Medicine, St. Louis, Missouri 63110
}

\begin{abstract}
Heparin cofactor II is a plasma protein that inhibits thrombin rapidly in the presence of either heparin or dermatan sulfate. We have determined the effects of two glycosaminoglycanbinding proteins, i.e., histidine-rich glycoprotein and platelet factor 4 , on these reactions. Inhibition of thrombin by heparin cofactor II and heparin was completely prevented by purified histidine-rich glycoprotein at the ratio of $13 \mu \mathrm{g}$ histidine-rich glycoprotein/ $\mu \mathrm{g}$ heparin. In contrast, histidine-rich glycoprotein had no effect on inhibition of thrombin by heparin cofactor II and dermatan sulfate at ratios of $\leq 128 \mu \mathrm{g}$ histidine-rich glycoprotein/ $\mu$ g dermatan sulfate. Removal of $85-90 \%$ of the histidine-rich glycoprotein from plasma resulted in a fourfold reduction in the amount of heparin required to prolong the thrombin clotting time from $14 \mathrm{~s}$ to $>180 \mathrm{~s}$ but had no effect on the amount of dermatan sulfate required for similar anticoagulant activity. In contrast to histidine-rich glycoprotein, purified platelet factor 4 prevented inhibition of thrombin by heparin cofactor II in the presence of either heparin or dermatan sulfate at the ratio of $2 \mu \mathrm{g}$ platelet factor $4 / \mu \mathrm{g}$ glycosaminoglycan. Furthermore, the supernatant medium from platelets treated with arachidonic acid to cause secretion of platelet factor 4 prevented inhibition of thrombin by heparin cofactor II in the presence of heparin or dermatan sulfate. We conclude that histidine-rich glycoprotein and platelet factor 4 can regulate the antithrombin activity of heparin cofactor II.
\end{abstract}

\section{Introduction}

Heparin cofactor II (HCII) ${ }^{1}$ is a glycoprotein in human plasma that rapidly inactivates thrombin in the presence of heparin or dermatan sulfate (1-7). HCII is similar to antithrombin III (ATIII) in structure and mechanism of action. Each inhibitor inactivates thrombin by forming an equimolar complex with the protease that is stable upon SDS polyacrylamide gel electrophoresis (4), the two inhibitors appear to have significant amino acid sequence homology in the region of the protease-

An abstract of this work has been published in Blood. 62:294. 1983. Address correspondence to Dr. Tollefsen.

Received for publication 8 August 1984 and in revised form 17 October 1984

1. Abbreviations used in this paper: ATIII, antithrombin III; CMcellulose, carboxymethylcellulose; Polybrene, 1,5-dimethyl-1,5-diazaundecamethylene polymethobromide; HCII, heparin cofactor II; HRG, histidine-rich glycoprotein; PF4, platelet factor 4.

J. Clin. Invest.

(C) The American Society for Clinical Investigation, Inc.

0021-9738/85/02/0496/06 \$1.00

Volume 75, February 1985, 496-501 binding site (8), and heparin binds to each inhibitor and thereby increases the rate of inhibition of thrombin $\sim 1,000$ fold $(4,9)$. However, HCII and ATIII differ in two important properties: $(a)$ whereas ATIII inhibits all of the serine proteases of the intrinsic coagulation system (10), the known protease specificity of $\mathrm{HCII}$ is restricted to thrombin $(2,4,6,7)$; and (b) dermatan sulfate greatly accelerates the reaction between thrombin and HCII but has no appreciable effect on the activity of ATIII (5).

ATIII appears to be involved in the prevention of intravascular coagulation, since patients with ATIII deficiency often develop recurrent thrombosis (11). The mechanism of action of ATIII in vivo has been difficult to study. However, recent experiments indicate that ATIII is activated within the microcirculation, perhaps by binding to heparin-like molecules on the luminal surface of endothelial cells $(12,13)$. The site of action of $\mathrm{HCII}$ is unknown, although current evidence suggests that it is not a major inhibitor of thrombin within the blood vessels (14). Nevertheless, HCII is potentially an important inhibitor of thrombin in the extravascular connective tissues where dermatan sulfate is prevalent (15).

Histidine-rich glycoprotein (HRG) in human plasma binds heparin and thereby prevents activation of ATIII in vitro (16). Similarly, platelet factor 4 (PF4) secreted from platelets during hemostasis binds heparin and neutralizes its anticoagulant effect $(17,18)$. The presence of a sufficient quantity of either of these proteins would therefore favor coagulation. In this study, we have investigated the ability of HRG and PF4 to affect the activation of $\mathrm{HCII}$ by heparin and dermatan sulfate. We have found that physiological concentrations of both proteins prevent activation of HCII by heparin and that PF4 also prevents activation of $\mathrm{HCII}$ by dermatan sulfate. In contrast, HRG has no effect on the ability of dermatan sulfate to activate $\mathrm{HCII}$.

\section{Methods}

Materials. Tosyl-Gly-Pro-Arg-p-nitroanilide (Chromozym TH) was obtained from Boehringer Mannheim, Indianapolis, IN; polyethylene glycol (Carbowax-6000) from Union Carbide, New York, NY; Polybrene from Aldrich Chemical Co., Milwaukee, WI; arachidonic acid from NuChek Prep, Elysian, MN; and dermatan sulfate (chondroitin sulfate B from porcine skin) from Sigma Chemical Co., St. Louis, MO. Dermatan sulfate was treated with $0.24 \mathrm{M} \mathrm{NaNO}_{2}$ in $1.8 \mathrm{M}$ acetic acid for $80 \mathrm{~min}$ at $22^{\circ} \mathrm{C}$ to degrade contaminating heparin (19) and dialyzed against water prior to use. Heparin (Panheprin, 1000 USP $\mathrm{U} / \mathrm{ml}$ ) was obtained from Abbott Laboratories, North Chicago, IL. Carboxymethylcellulose (CM-cellulose) (Cellex CM) was obtained from Bio-Rad Laboratories, Richmond, CA.

Proteins. Human thrombin, HCII, and ATIII were purified and assayed as previously described (4). The concentrations of these proteins were determined from the absorbance at $280 \mathrm{~nm}$ assuming extinction coefficients $(1 \mathrm{mg} / \mathrm{ml})$ of $1.83(20), 1.17(4)$, and $0.57(21)$, respectively. 
Purified human HRG $\left(M_{\mathrm{r}}=75,000\right.$; ref. 16) was a gift from Drs. H. R. Lijnen and D. Collen, University of Leuven, Belgium. Purified human PF4 (22) was provided by Drs. J. Huang, S. Huang, and T. Deuel, Washington University, St. Louis, MO. Hirudin (23) was purchased from Sigma Chemical Co.

Inhibition of thrombin by HCII and ATIII. Incubations contained the inhibitor, the glycosaminoglycan, and other reagents at the final concentrations indicated in $100 \mu \mathrm{l}$ of $0.15 \mathrm{M} \mathrm{NaCl}, 0.02 \mathrm{M}$ Tris- $\mathrm{HCl}$, $1 \mathrm{mg} / \mathrm{ml}$ polyethylene glycol, $\mathrm{pH}$ 7.4. Reactions were initiated by addition of thrombin. At specified times, $100 \mu \mathrm{l}$ of a solution containing $0.1 \mathrm{mM}$ tosyl-Gly-Pro-Arg-p-nitroanilide and $0.05 \mathrm{mg} / \mathrm{ml}$ 1,5-dimethyl1,5-diazaundecamethylene polymethobromide (Polybrene) in water was added. Hydrolysis of the substrate was stopped after exactly $120 \mathrm{~s}$ by addition of $10 \mu \mathrm{l}$ of hirudin $(200 \mathrm{U} / \mathrm{ml})$, and the absorbance at 405 $\mathrm{nm}$ was determined (5).

The incubations in Figs. 1, 3, and 4, and Tables I and II contained a molar excess of HCII compared to thrombin, resulting in time courses of inhibition that approximated pseudo-first order kinetics (4, 5). Heparin and dermatan sulfate were employed at relatively low concentrations, which yielded $\sim 100$-fold and $\sim 16$-fold enhancement of the rate of inhibition of thrombin, respectively. Maximum rate enhanements of 800 -fold and 1300 -fold have been observed with higher concentrations of heparin and dermatan sulfate (5). Under the experimental conditions, the extent of inhibition of thrombin decreased linearly as the glycosaminoglycan concentration was decreased. No appreciable inhibition occurred in controls in which the glycosaminoglycan was omitted. For convenience, reaction times of $20 \mathrm{~s}$ for heparin and $120 \mathrm{~s}$ for dermatan sulfate were chosen to produce $\sim 50 \%$ inhibition of thrombin in the absence of HRG or PF4.

Removal of $H R G$ from plasma. Human venous blood $(4.5 \mathrm{ml})$ was collected into evacuated tubes containing $0.5 \mathrm{ml}$ of $0.129 \mathrm{M}$ buffered sodium citrate (Vacutainer 6418, Becton-Dickinson, Rutherford, NJ). After removal of the cells by centrifugation, the plasma was absorbed with CM-cellulose by the procedure of Heimburger et al. (24). The CM-cellulose was cycled according to the manufacturer and suspended in distilled water adjusted to $\mathrm{pH}$ 6.0. A portion of the suspension containing $0.4 \mathrm{~g}$ (dry weight) of the resin was centrifuged, and the supernatant solution was decanted. The resin was resuspended in 10 $\mathrm{ml}$ of plasma (total volume $\sim 13 \mathrm{ml}$ ) and mixed for $1 \mathrm{~h}$ at $22^{\circ} \mathrm{C}$. The resin was removed by centrifugation, and the $\mathrm{pH}$ of the supernatant plasma was adjusted to 7.0 with $1 \mathrm{M} \mathrm{NaOH}$. HRG was measured in the absorbed plasma by the Laurell immunoelectrophoresis technique (25) using monospecific rabbit anti-HRG antiserum provided by Dr. H. R. Lijnen and Dr. D. Collen (16).

Determination of the thrombin clotting time. $200 \mu \mathrm{l}$ of plasma was mixed with $50 \mu \mathrm{l}$ of dermatan sulfate or heparin in $0.15 \mathrm{M} \mathrm{NaCl}, 6.6$ $\mathrm{mg} / \mathrm{ml}$ polyethylene glycol, $10 \mathrm{mM} \mathrm{CaCl}$, and $10 \mathrm{mM}$ imidazole$\mathrm{HCl}, \mathrm{pH}$ 7.4. The mixture was warmed to $37^{\circ} \mathrm{C}, 50 \mu \mathrm{l}$ of thrombin ( $\sim 40 \mathrm{nM}$ in the above buffer) was added, and the clotting time was determined with a Fibrometer (Becton-Dickinson Co.).

Treatment of platelets with arachidonic acid. Human platelets were washed and resuspended in $0.14 \mathrm{M} \mathrm{NaCl}, 0.015 \mathrm{M}$ Tris- $\mathrm{HCl}, 0.0055$ $\mathrm{M}$ glucose, $\mathrm{pH} 7.5$, at a final concentration of $1.5 \times 10^{9}$ platelets $/ \mathrm{ml}$ (26). $1 \mathrm{ml}$ of the platelet suspension was incubated for $10 \mathrm{~min}$ at $22^{\circ} \mathrm{C}$ with $100 \mu \mathrm{l}$ of $0.25 \mathrm{mM}$ arachidonic acid dissolved in $50 \mathrm{mM}$ $\mathrm{NaHCO}_{3}$. The platelets were then removed by centrifugation for 10 min in an Eppendorf microcentrifuge. Control medium was prepared by incubating $1 \mathrm{ml}$ of platelets with $100 \mu \mathrm{l}$ of $50 \mathrm{mM} \mathrm{NaHCO}$ without arachidonic acid. The concentration of PF4 in the medium was determined with a commercial radioimmunoassay kit (Abbott Laboratories).

\section{Results}

HRG blocks activation of HCII by heparin but not by dermatan sulfate. The normal concentration of HRG in plasma is $\sim 1.8$ $\mu \mathrm{M}(135 \mu \mathrm{g} / \mathrm{ml})$ (16). Fig. 1 shows the effect of $0-3.4 \mu \mathrm{M}$

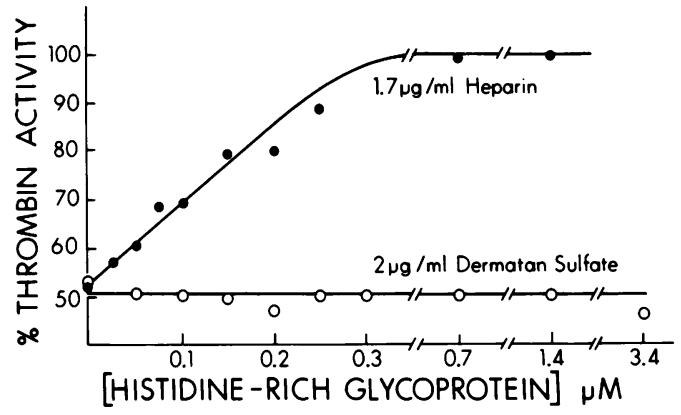

Figure 1. Effect of HRG on HCII activity in the presence of heparin or dermatan sulfate. HCII ( $38 \mathrm{nM})$, thrombin $(2 \mathrm{nM})$, and varying concentrations of purified HRG were allowed to react for $20 \mathrm{~s}$ in the presence of $1.7 \mu \mathrm{g} / \mathrm{ml}$ heparin $(\bullet)$ or $120 \mathrm{~s}$ in the presence of $2.0 \mu \mathrm{g} /$ $\mathrm{ml}$ dermatan sulfate (o). Residual thrombin activity was then determined by hydrolysis of the chromogenic substrate tosyl-Gly-Pro-Argp-nitroanilide as described in Methods.

HRG on the extent of inhibition of thrombin by HCII. In the presence of $1.7 \mu \mathrm{g} / \mathrm{ml}$ heparin, inhibition of thrombin by $\mathrm{HCII}$ is completely prevented by HRG at concentrations $\geq 0.7 \mu \mathrm{M}$ (Fig. 1, closed circles). At concentrations of HRG between 0 and $0.25 \mu \mathrm{M}$, the amount of thrombin activity remaining in the incubation varies linearly with the concentration of HRG. Extrapolation of the linear part of the curve to $100 \%$ thrombin activity indicates that $0.3 \mu \mathrm{M}$ HRG is required to neutralize $1.7 \mu \mathrm{g} / \mathrm{ml}$ heparin (i.e., $13 \mu \mathrm{g} \mathrm{HRG} / \mu \mathrm{g}$ heparin). As previously reported for systems containing ATIII and heparin (16), 2.5 $\mu \mathrm{M}$ EDTA abolishes the effect of HRG and thus allows inhibition of thrombin by HCII and heparin to occur (Table I). This effect may be due to removal of a divalent cation from HRG or to a direct interaction between HRG and EDTA.

In contrast, $\mathrm{HRG}$ at concentrations as high as $3.4 \mu \mathrm{M}$ has no effect on inhibition of thrombin by $\mathrm{HCII}$ in the presence of $2.0 \mu \mathrm{g} / \mathrm{ml}$ dermatan sulfate (Fig. 1, open circles). This result suggests that HRG does not bind to dermatan sulfate or, alternatively, that dermatan sulfate molecules with HRG bound to them remain able to activate HCII. To determine whether dermatan sulfate binds HRG and thereby prevents the protein

Table I. Effect of EDTA on the Neutralization of Heparin by HRG

\begin{tabular}{lcc}
\hline HRG & EDTA & Thrombin activity \\
\hline$\mu M$ & $\mu M$ & $\%$ \\
0 & 0 & 53 \\
& 10 & 60 \\
0.27 & 0 & 100 \\
& 0.5 & 99 \\
& 1.0 & 85 \\
& 2.5 & 61 \\
& 5 & 59
\end{tabular}

Each incubation contained thrombin ( $2 \mathrm{nM}), \mathrm{HCII}(38 \mathrm{nM})$, and heparin $(0.67 \mu \mathrm{g} / \mathrm{ml})$. In addition, HRG and EDTA were present at the final concentrations indicated. Residual thrombin activity was determined after a 20-s incubation as described in Methods. 
from binding to heparin, we studied the effect of a high concentration of dermatan sulfate on the ability of HRG to neutralize heparin activity in a system containing purified ATIII and thrombin. The control experiments shown in Table II indicate that dermatan sulfate does not activate ATIII (cf. lines 1, 2, and 7) nor does it protect thrombin from inhibition by ATIII in the presence of heparin (cf. lines 3 and 6). The data in Table II indicate that $0.27 \mu \mathrm{M}$ HRG reduces the inhibition of thrombin from $54 \%$ (line 3 ) to $7 \%$ (line 4 ) in incubations with ATIII and $0.67 \mu \mathrm{g} / \mathrm{ml}$ heparin. Inclusion of $100 \mu \mathrm{g} / \mathrm{ml}$ of dermatan sulfate in the incubation does not alter the effect of HRG (cf. lines 4 and 5). These findings indicate that dermatan sulfate does not compete effectively with heparin for binding to HRG.

HRG does not affect the ability of dermatan sulfate to prolong the clotting time of plasma. The results in Fig. 1 lead to the prediction that variations in the concentration of HRG in plasma should have no effect on the anticoagulant activity of dermatan sulfate. HRG-depleted plasma was prepared by absorption with CM-cellulose as described in Methods. This procedure removed $85-90 \%$ of the HRG as assessed by rocket immunoelectrophoresis (Fig. 2). Table III indicates that the clotting time of plasma after addition of thrombin is prolonged from $\sim 14 \mathrm{~s}$ to $>180 \mathrm{~s}$ by $4.2 \mu \mathrm{g} / \mathrm{ml}$ dermatan sulfate. Neither absorption of plasma with CM-cellulose nor reconstitution of the absorbed plasma with purified HRG significantly changes the concentration of dermatan sulfate required to prolong the clotting time. In contrast, absorption of plasma with CMcellulose results in a fourfold reduction in the amount of heparin required for anticoagulation. As noted previously (16), depletion of HRG is responsible for this effect, since reconstitution of the plasma with purified HRG restores the requirement for the higher heparin concentration.

PF4 blocks activation of HCII by both heparin and dermatan sulfate. Fig. 3 shows the effect of 0-7.7 $\mu \mathrm{M}$ PF4 $(0-60 \mu \mathrm{g} / \mathrm{ml})$ on the extent of inhibition of thrombin by $\mathrm{HCII}$ in the

Table II. Neutralization of Heparin by $H R G$ in the Presence of Excess Dermatan Sulfate

\begin{tabular}{lll}
\hline Experiment & Additions & Thrombin activity \\
\hline & & $\%$ \\
1 & Thrombin & 100 \\
2 & Thrombin + ATIII & 100 \\
3 & Thrombin + ATIII + heparin & 46 \\
4 & Thrombin + ATIII + heparin & 93 \\
& $\quad+$ HRG & \\
5 & Thrombin + ATIII + heparin & 95 \\
6 & $\quad+$ HRG + dermatan sulfate & \\
& Thrombin + ATIII + heparin & 49 \\
7 & $\quad+$ dermatan sulfate & 102 \\
& Thrombin + ATIII & \\
& + dermatan sulfate
\end{tabular}

Incubations contained various combinations of reagents at the following final concentrations: thrombin, $2 \mathrm{nM}$; ATIII, $10 \mathrm{nM}$; HRG, 0.27 $\mu \mathrm{M}$; heparin, $0.67 \mu \mathrm{g} / \mathrm{ml}$; and dermatan sulfate, $100 \mu \mathrm{g} / \mathrm{ml}$. Residual thrombin activity was determined after a 20-S incubation as described in Methods.

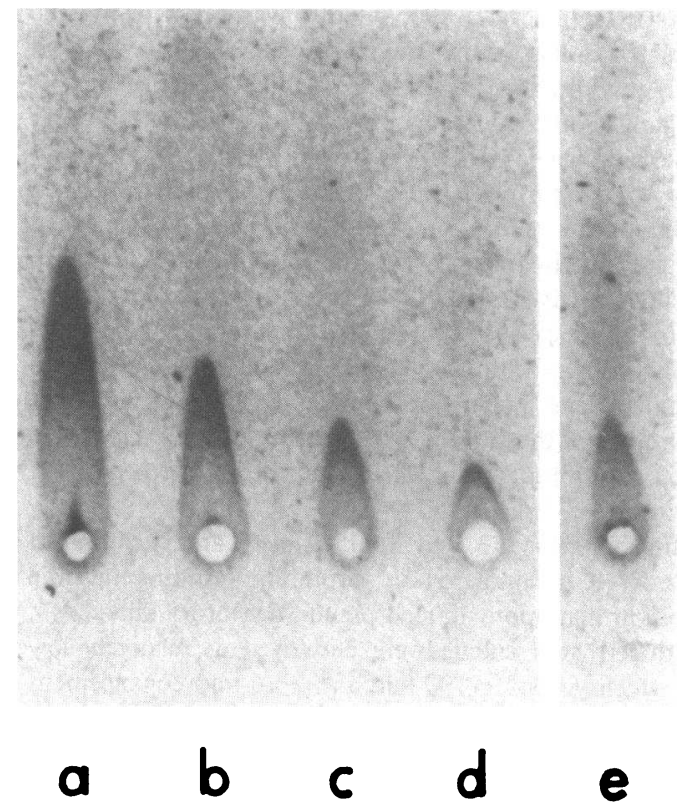

Figure 2. Immunoelectrophoresis of HRG-depleted plasma. Citrateanticoagulated human plasma was absorbed with CM-cellulose as described in Methods. The plasma was then tested for HRG antigen by the Laurell immunoelectrophoresis technique. Lanes a-d contain $5 \mu \mathrm{l}$ of control plasma diluted $1: 2,1: 4,1: 8$, and 1:16, respectively. Lane e contains $5 \mu \mathrm{l}$ of undiluted HRG-depleted plasma.

presence of $1.7 \mu \mathrm{g} / \mathrm{ml}$ heparin or $2.5 \mu \mathrm{g} / \mathrm{ml}$ dermatan sulfate. Inhibition of thrombin by $\mathrm{HCII}$ in the presence of either glycosaminoglycan is prevented by PF4 at concentrations $\geq 1.3$ $\mu \mathrm{M}$. Extrapolations of the linear parts of the curves to $100 \%$ thrombin activity suggest that $0.45 \mu \mathrm{M}$ PF4 is required to neutralize $1.7 \mu \mathrm{g} / \mathrm{ml}$ heparin (i.e., $2 \mu \mathrm{g}$ PF4/ $\mu \mathrm{g}$ heparin) and that $0.64 \mu \mathrm{M}$ PF4 is required to neutralize $2.5 \mu \mathrm{g} / \mathrm{ml}$ dermatan sulfate (i.e., $2 \mu \mathrm{g} \mathrm{PF} 4 / \mu \mathrm{g}$ dermatan sulfate).

PF4 is secreted from platelets bound to a proteoglycan carrier molecule (27. 28). To determine whether the proteoglycan interferes with the interaction between PF4 and dermatan sulfate shown in Fig. 3, we prepared supernatant media from washed human platelets treated with arachidonic acid to cause secretion as well as from control platelets incubated without arachidonic acid. Fig. 4 indicates the effects of various amounts of the supernatant media on the extent of inhibition of thrombin by HCII in the presence of heparin (open symbols) or dermatan sulfate (closed symbols). Final concentrations of secreted PF4 $\geq 0.3 \mu \mathrm{M}$ prevent inhibition of thrombin in the presence of either glycosaminoglycan. The amounts of PF4 required to neutralize the glycosaminoglycans in this experiment, i.e., $1.4 \mu \mathrm{g} \mathrm{PF} 4 / \mu \mathrm{g}$ heparin and $1.2 \mu \mathrm{g} \mathrm{PF4/ \mu g} \mathrm{dermatan}$ sulfate, are similar to the amounts determined in the experiment with purified PF4 (Fig. 3).

\section{Discussion}

Dermatan sulfate, heparin, and certain heparan sulfate preparations are able to activate $\mathrm{HCII}$, i.e., to markedly increase the rate of complex formation between thrombin and the inhibitor protein (5). In contrast, while heparin and heparan sulfate 
Table III. Prolongation of the Thrombin Clotting Time of Plasma by Dermatan Sulfate and Heparin

\begin{tabular}{|c|c|c|c|c|c|c|c|c|c|c|c|}
\hline \multirow[b]{2}{*}{ Plasma } & \multicolumn{6}{|c|}{ Final [dermatan sulfate] $(\mu \mathrm{g} / \mathrm{ml})$} & \multicolumn{5}{|c|}{ Final [heparin] $(\mu \mathrm{g} / \mathrm{ml})$} \\
\hline & 0 & 0.52 & 1.0 & 2.1 & 4.2 & 8.3 & 0.21 & 0.42 & 0.85 & 1.7 & 3.4 \\
\hline \multicolumn{12}{|l|}{ Clotting time $(s)$} \\
\hline Control & 14.0 & 15.4 & 16.4 & 24.3 & $>180$ & $>180$ & 15.9 & 20.4 & 31.8 & $>180$ & $>180$ \\
\hline HRG-depleted & 14.4 & 16.9 & 20.4 & 33.4 & $>180$ & $>180$ & 20.4 & $>180$ & $>180$ & $>180$ & $>180$ \\
\hline HRG-reconstituted & 14.0 & & & 29.6 & $>180$ & $>180$ & & 15.9 & 21.4 & $>180$ & \\
\hline
\end{tabular}

HRG was absorbed from plasma by incubation with CM-cellulose as described in Methods. The final [HRG] was 10-15\% of the starting concentration. The plasma was reconstituted by addition of purified HRG to give the original concentration $(\sim 1.8 \mu \mathrm{M})$. Clotting times after addition of thrombin were determined as described in Methods.

activate ATIII, dermatan sulfate is inactive. Other glycosaminoglycans, including chondroitin-4-sulfate, chondroitin-6-sulfate, hyaluronate, and keratan sulfate, are inactive with both inhibitors.

It appears that specific oligosaccharide sequences in dermatan sulfate and heparin are necessary for activity with HCII. Griffith and Marbet have shown that molecules of either glycosaminoglycan that bind to a column of immobilized HCII have higher activities than molecules that do not bind (9). In both cases, the high-affinity fraction constitutes a minority of the molecules in the initial glycosaminoglycan preparation. Based on experiments in which heparin was sequentially fractionated according to charge density and binding affinity for ATIII, Hurst et al. have suggested that HCII and ATIII interact with different heparin species (29). However, the structure of the HCII-binding domain of heparin is unknown. Recently, we have prepared oligosaccharides of dermatan sulfate by partial chemical depolymerization and have found that the smallest fragment that binds HCII contains 8 monosaccharide units (30). The structure of the HCII-binding octasaccharide is currently under investigation.

In contrast to the inhibitor proteins, which bind only a subpopulation of heparin molecules, HRG and PF4 can bind

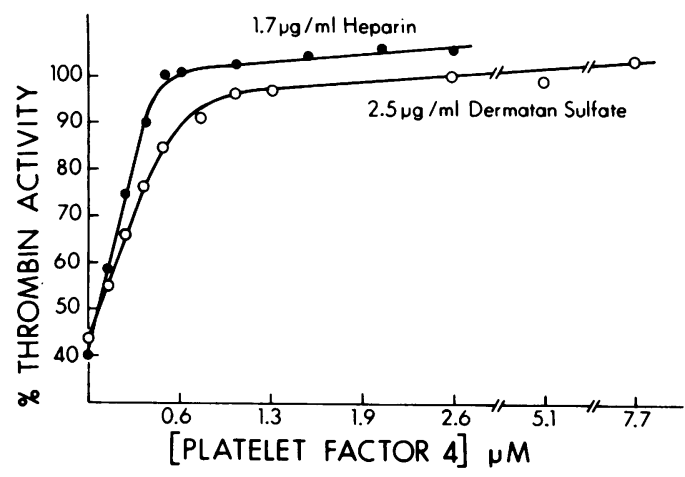

Figure 3. Effect of PF4 on HCII activity in the presence of heparin or dermatan sulfate. $\mathrm{HCII}(38 \mathrm{nM})$, thrombin $(2 \mathrm{nM})$, and varying concentrations of purified PF4 were allowed to react for $20 \mathrm{~s}$ in the presence of $1.7 \mu \mathrm{g} / \mathrm{ml}$ heparin $(\bullet)$ or for $120 \mathrm{~s}$ in the presence of 2.5 $\mu \mathrm{g} / \mathrm{ml}$ dermatan sulfate (o). Residual thrombin activity was then determined by hydrolysis of tosyl-Gly-Pro-Arg-p-nitroanilide as described in Methods. heparin fractions with both high and low affinity for ATIII $(16,31)$. Both HRG and PF4 block the catalytic effect of heparin on the thrombin-ATIII reaction. However, it is unknown whether the primary effects of HRG and PF4 are to prevent binding of heparin to ATIII or, alternatively, to prevent formation of a ternary complex among ATIII, heparin, and the protease.

A major finding of the present investigation is that $H R G$ prevents the activation of $\mathrm{HCII}$ by heparin but not by dermatan sulfate. The apparent stoichiometry of the interaction between HRG and heparin determined from Fig. 1 is consistent with binding of $1 \mathrm{HRG}$ molecule/20-22 monosaccharide units of heparin. In contrast, HRG at approximately twice the concentration present in plasma $(1.8 \mu \mathrm{M})$ has no appreciable effect on the activation of HCII by dermatan sulfate (Fig. 1). This result in combination with the data in Table II, which show that excess dermatan sulfate does not block the effect of HRG

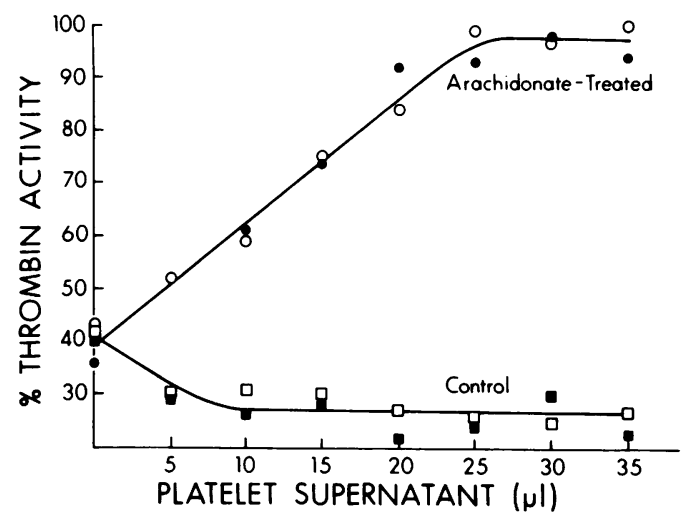

Figure 4. Effect of platelet supernatant medium on the activity of HCII. Washed human platelets $\left(1.5 \times 10^{9} / \mathrm{ml}\right)$ were incubated with $25 \mu \mathrm{M}$ arachidonic acid for $10 \mathrm{~min}$ (see Methods). Varying amounts of the supernatant medium were then incubated with $\mathrm{HCII}(38 \mathrm{nM})$, thrombin $(2 \mathrm{nM})$, and $1.7 \mu \mathrm{g} / \mathrm{ml}$ heparin (O) or $2.0 \mu \mathrm{g} / \mathrm{ml}$ dermatan sulfate (๑) in a final volume of $100 \mu \mathrm{l}$ as described in Fig. 1. Parallel incubations containing the supernatant medium from untreated platelets in addition to thrombin, HCII, and heparin ( $\square$ ) or dermatan sulfate ( $\square$ ) were also carried out. The media from treated and untreated platelets contained $1.2 \mu \mathrm{M}$ and $<0.1 \mu \mathrm{M}$ PF4, respectively, by radioimmunoassay. Residual thrombin activity was determined by hydrolysis of tosyl-Gly-Pro-Arg-p-nitroanilide. 
on heparin, suggest that HRG binds weakly, if at all, to dermatan sulfate. On the other hand, PF4 prevents activation of HCII by both heparin and dermatan sulfate (Figs. 3 and 4). The apparent stoichiometries of the interactions with PF4 determined from Figs. 3 and 4 are very similar for both heparin and dermatan sulfate and are consistent with binding of 1 PF4 monomer/14-22 monosaccharide units.

The experiments shown in Table III and Fig. 4 were performed in an attempt to more closely approximate conditions that might be present in vivo. The data in Table III indicate that removal of $85-90 \%$ of the HRG from plasma does not affect the concentration of dermatan sulfate required to prolong the clotting time after addition of exogenous thrombin. This result is consistent with the experiment in Fig. 1 , because $\mathrm{HCII}$ is the major inhibitor of thrombin in the presence of dermatan sulfate (5). PF4 is secreted from platelets as a tetramer (monomer $M_{\mathrm{r}}=7800$ ) bound to a platelet proteoglycan containing principally chondroitin-4-sulfate (27, 28). After secretion, PF4 can dissociate from the carrier proteoglycan and bind to other glycoaminoglycans. The relative binding affinities of PF4 to the various glycosaminoglycans are reported to be as follows: heparin $>$ dermatan sulfate $>$ chondroitin-6-sulfate $>$ chondroitin-4-sulfate (17). Fig. 4 suggests that the carrier proteoglycan does not prevent binding of PF4 to dermatan sulfate, since activation of $\mathrm{HCII}$ by dermatan sulfate was prevented by supernatant medium from stimulated platelets. We have not investigated the possibility that other secreted proteins which bind heparin with lower affinity than does PF4 (e.g., low-affinity PF4, $\beta$-thromboglobulin, and thrombospondin) $(32,33)$ effectively compete with $\mathrm{HCII}$ for binding to dermatan sulfate.

Although HCII is a very potent inhibitor of thrombin in vitro, its physiological function is unknown. When thrombin is injected intravenously into experimental animals or perfused with plasma through a microvascular preparation, it is primarily inhibited by ATIII $(12,14)$. This suggests that the normal role of HCII is not to inhibit intravascular thrombin. Nevertheless, dermatan sulfate given intravenously prevents formation of thrombi in rabbits, apparently by activating HCII within the blood vessels (34). Physiologically, however, HCII may inhibit thrombin in extravascular sites where dermatan sulfate is prevalent and thereby regulate activities of the protease such as mitogenesis (35) or chemotaxis (36). Modulation of the activity of HCII by PF4 may be important in controlling these processes. After vessel injury, platelets may secrete PF4 into the extravascular space where it can bind dermatan sulfate and allow thrombin to remain active even in the presence of HCII. In the absence of platelet degranulation, or after clearance of PF4 from the tissues, thrombin may be inactivated by HCII even though HRG may be present concomitantly in the extravascular space.

\section{Acknowledgments}

We are grateful to Drs. H. R. Lijnen, D. Collen, J. Huang, S. Huang, and T. Deuel for providing reagents for these studies, and to Mrs. Lois Isenberg for preparing the manuscript.

Dr. Tollefsen is the recipient of Research Career Development Award HL-01079 from the National Institutes of Health. These studies were supported by a grant from the National Institutes of Health (HL-27589).

\section{References}

1. Briginshaw, G. F., and J. N. Shanberge. 1974. Identification of two distinct heparin cofactors in human plasma. Separation and partial purification. Arch. Biochem. Biophys. 161:683-690.

2. Briginshaw, G. F., and J. N. Shanberge. 1974. Identification of two distinct heparin cofactors in human plasma: II. Inhibition of thrombin and activated factor X. Thromb. Res. 4:463-477.

3. Tollefsen, D. M., and M. K. Blank. 1981. Detection of a new heparin-dependent inhibitor of thrombin in human plasma. J. Clin. Invest. 68:589-596.

4. Tollefsen, D. M., P. W. Majerus, and M. K. Blank. 1982. Heparin cofactor II. Purification and properties of a heparin-dependent inhibitor of thrombin in human plasma. J. Biol. Chem. 257:21622169.

5. Tollefsen, D. M., C. A. Pestka, and W. J. Monafo. 1983. Activation of heparin cofactor II by dermatan sulfate. J. Biol. Chem. 258:6713-6716.

6. Griffith, M. J., T. Carraway, G. C. White, and F. A. Dombrose. 1983. Heparin cofactor activities in a family with hereditary antithrombin III deficiency. Evidence for a second heparin cofactor in human plasma. Blood. 61:111-118.

7. Wunderwald, P., W. J. Schrenk, and H. Port. 1982. Antithrombin BM from human plasma. An antithrombin binding moderately to heparin. Thromb. Res. 25:177-191.

8. Griffith, M. J., C. M. Noyes, N. Allen, and G. B. Villanueva. 1984. Evidence for structurally similar regions in antithrombin III and heparin cofactor II. Fed. Proc. 43:1963. (Abstr.)

9. Griffith, M. J., and G. A. Marbet. 1983. Dermatan sulfate and heparin can be fractionated by affinity for heparin cofactor II. Biochem. Biophys. Res. Commun. 112:663-670.

10. Rosenberg, R. D. 1977. Biologic actions of heparin. Semin. Hematol. 14:427-439.

11. Harpel, P. C. 1982. Blood proteolytic enzyme inhibitors: their role in modulating blood coagulation and fibrinolytic enzyme pathways. In Hemostasis and Thrombosis, R. W. Colman, J. Hirsh, V. J. Marder, and E. W. Salzman, editors. J. B. Lippincott Co., Philadelphia, pp. 738-747.

12. Busch, C., and W. G. Owen. 1982. Identification in vitro of an endothelial cell surface cofactor for antithrombin III. Parallel studies with isolated perfused rat hearts and microcarrier cultures of bovine endothelium. J. Clin. Invest. 69:726-729.

13. Marcum, J. A., L. Fritze, S. J. Galli, G. Karp, and R. D. Rosenberg. 1983. Microvascular heparinlike species with anticoagulant activity. Am. J. Physiol. 245:H725-H733.

14. MacIntosh, S., H. Jakubowski, and W. G. Owen. 1984. Regulation of clearance and inhibition of intra-vascular thrombin. Fed. Proc. 43:1946. (Abstr.)

15. Lindahl, U., and M. Höök. 1978. Glycosaminoglycans and their binding to biological macromolecules. Annu. Rev. Biochem. 47:385-417.

16. Lijnen, H. R., M. Hoylaerts, and D. Collen. 1983. Heparinbinding properties of human histidine-rich glycoprotein. Mechanism and role in the neutralization of heparin in plasma. J. Biol. Chem. 258:3803-3808.

17. Handin, R. I., and H. J. Cohen. 1976. Purification and binding properties of human platelet factor four. J. Biol. Chem. 251:42734282.

18. Levine, S. P., and H. Wohl. 1976. Human platelet factor 4: purification and characterization by affinity chromatography. J. Biol. Chem. 251:324-328.

19. Teien, A. N., U. Abildgaard, and M. Höök. 1976. The anticoagulant effect of heparan sulfate and dermatan sulfate. Thromb. Res. 8:859-867.

20. Fenton, J. W., II, M. J. Fasco, A. B. Stackrow, D. L. Aronson, A. M. Young, and J. S. Finlayson. 1977. Human thrombins: production, 
evaluation, and properties of $\alpha$-thrombin. J. Biol. Chem. 252:35873598.

21. Kurachi, K., G. Schmer, M. A. Hermodson, D. C. Teller, and E. W. Davie. 1976. Characterization of human, bovine, and horse antithrombin III. Biochemistry. 15:368-373.

22. Deuel, T. F., P. S. Keim, M. Farmer, and R. L. Heinrikson. 1977. Amino acid sequence of human platelet factor 4. Proc. Natl. Acad. Sci. USA. 74:2256-2258.

23. Bagdy, D., E. Barabas, L. Gráf, T. E. Peterson, and S. Magnusson. 1976. Hirudin. Methods Enzymol. 45:669-678.

24. Heimburger, N., H. Haupt, T. Kranz, and S. Baudner. 1972. Humanserumproteine mit hoher affinitat zu carboxymethylcellulose, II. Hoppe-Seyler's Z. Physiol. Chem. 353:1133-1140.

25. Laurell, C.-B. 1966. Quantitative estimation of proteins by electrophoresis in agarose gel containing antibodies. Anal. Biochem. 15:45-52.

26. Tollefsen, D. M., J. R. Feagler, and P. W. Majerus. 1974. Induction of the platelet release reaction by phytohemagglutinin. $J$. Clin. Invest. 53:211-218.

27. Barber, A. J., R. Käser-Glanzmann, M. Jakábová, and E. F. Lüscher. 1972. Characterization of a chondroitin-4-sulfate proteoglycan carrier for heparin neutralizing activity (platelet factor 4 ) released from human blood platelets. Biochim. Biophys. Acta. 286:312-329.

28. Huang, S. S., J. S. Huang, and T. F. Deuel. 1982. Proteoglycan carrier of human platelet factor 4 . Isolation and characterization. $J$. Biol. Chem. 257:11546-11550.
29. Hurst, R. E., M.-C. Poon, and M. J. Griffith. 1983. Structureactivity relationships of heparin. Independence of heparin charge density and antithrombin-binding domains in thrombin inhibition by antithrombin and heparin cofactor II. J. Clin. Invest. 72:1042-1045.

30. Tollefsen, D. M., M. E. Peacock, and W. J. Monafo. 1984. Molecular size of dermatan sulfate oligosaccharides required to bind and activate heparin cofactor II. Fed. Proc. 43:1843. (Abstr.)

31. Bock, P. E., M. Luscombe, S. E. Marshall, D. S. Pepper, and J. J. Holbrook. 1980. The multiple complexes formed by the interaction of platelet factor 4 with heparin. Biochem. J. 191:769-776.

32. Paul, D., S. Niewiarowski, K. G. Varma, B. Rucinski, S. Rucker, and E. Lange. 1980. Human platelet basic protein association with antiheparin and mitogenic activities: purification and partial characterization. Proc. Natl. Acad. Sci. USA. 77:5914-5918.

33. Lawler, J. W., H. S. Slayter, and J. E. Coligan. 1978. Isolation and characterization of a high molecular weight glycoprotein from human blood platelets. J. Biol. Chem. 253:8609-8616.

34. Buchanan, M. R., B. Boneu, F. Ofosu, and J. Hirsh. 1984. The relative importance of thrombin inhibition and factor $\mathrm{Xa}$ inhibition to the antithrombotic effects of heparin. Blood. In press.

35. Chen, L. B., and J. M. Buchanan. 1975. Mitogenic activity of blood components. I. Thrombin and prothrombin. Proc. Natl. Acad. Sci. USA. 72:131-135.

36. Bar-Shavit, R., A. Kahn, M. S. Mudd, G. D. Wilner, K. G. Mann, and J. W. Fenton, II. 1984. Localization of a chemotactic domain in human thrombin. Biochemistry. 23:397-400. 